\title{
Comparative Evaluation of Different Emulators for Cardiac Mechanics
}

\author{
David Dalton ${ }^{1}$, Alan Lazarus ${ }^{1}$, Dirk Husmeier ${ }^{1}$ \\ ${ }^{1}$ School of Mathematics and Statistics, University of Glasgow, \\ Glasgow G12 8SQ, UK \\ d.dalton.1@ research.gla.ac.uk; a.lazarus.1@research.gla.ac.uk; dirk.husmeier@glasgow.ac.uk
}

\begin{abstract}
This paper outlines a comparison of different emulation based approaches to the task of parameter inference in a biomechanical model of the left ventricle of the heart, where the emulation models can account for variations in left ventricle geometry. Models considered include Gaussian processes, neural networks and random forests. We are able to achieve accurate parameter estimation for two of the model parameters, while the extension of statistical emulation to the multi geometry case allows us to observe identifiability issues in some of the model parameters. This was not observed in our previous single geometry emulation studies. Overall, this study shows the ability to generalize the single geometry emulation strategy to multiple geometries, pushing us closer towards in clinic decision support systems.
\end{abstract}

Keywords: Holzapfel Ogden Model, Statistical Emulation, Gaussian Processes, Neural Networks.

\section{Introduction}

Recent advances into differential-equation based models of cardiac mechanics have demonstrated the potential for their use in a clinical setting for the diagnosis of cardiac defects [i]]. One such model is the Holzapfel-Ogden (HO) constitutive law [2]. The HO law describes the deformations and stress-strain behaviour of the left-ventricle heart during diastole, given stiffness levels in the cardiac tissue. Finding these stiffness parameter values is of clinical interest to help detect problems in cardiac behaviour, however they cannot be directly measured by non-invasive means. One possible, non-invasive approach to determining the parameters is to make use of Magnetic Resonance Imaging (MRI) scans to measure the beginning and end diastolic states of the LV. We can then estimate the stiffness levels as those values which minimise the difference between the quantities extracted from the MRI, and those predicted by the model. In a proof of concept study [3], it was demonstrated that this approach led to an improved and clinically relevant understanding of cardiac function. Unfortunately, calibrating the physiological parameters in this manner is extremely computationally expensive, as the system of partial differential equations that describe the mechanics of the LV have no closed form solutions. Instead, numerical procedures based on finite element discretisation are required, which can take several minutes to converge on a high performance computer. Since a typical parameter optimisation may require this process to be iterated hundreds or thousands of times, it is rendered unsuitable as a real-time clinical decision support tool.

One method which can be used to overcome these computational costs is emulation [4]. In emulation, we approximate the simulator with a statistical surrogate model called an emulator, which is trained on a number of evaluations from the simulator. While generating these simulations is extremely computationally costly, all of the computations can be done in advance of clinical deployment. In clinic, fast parameter estimates for a patient can then be made by minimising the discrepancy between the quantities measured by MRI and the corresponding values predicted by the computationally cheap emulator.

This work compares different methods, including Gaussian processes, neural networks and random forests, on the task of emulating the HO model which allows for a subject's specific LV geometry to be accounted for. This builds on earlier, proof of concept work [5, 6], which demonstrated the effectiveness of emulation for a fixed LV geometry. Accounting for subject-specific variations in LV geometry is crucial for practical applications. We compare the parameter estimation accuracy of each emulation approach on a test set of independent samples simulated from the model, for which the true parameter values are known.

\section{The Holzapfel-Ogden Law}

The HO law models the dynamics of the muscle tissue of the LV, known as the myocardium, during diastole. Diastole is the passive period of the cardiac cycle during which the heart fills with blood. To simulate the diastolic behaviour of a given $\mathrm{LV}$, the model requires two primary inputs. The first of these is a finite element reconstruction of the LV geometry, which 
we denote $\mathcal{H}$. Patient-specific reconstructions can be generated in real-time from MRI scans, the details of which have been previously reported [5]. Secondly, we must specify the material parameter vector

$$
\xi=\left(a, b, a_{\mathrm{f}}, b_{\mathrm{f}}, a_{\mathrm{s}}, b_{\mathrm{s}}, a_{\mathrm{fs}}, b_{\mathrm{fs}}\right)
$$

where $a$ and $b$ are the isotropic stiffness of the tissue, $a_{\mathrm{f}}$ and $b_{\mathrm{f}}$ are the values of the tissue stiffness in the fiber direction, $a_{\mathrm{s}}$ and $b_{\mathrm{s}}$ are the sheet direction stiffness values, while $a_{\mathrm{fs}}$ and $b_{\mathrm{fs}}$ describe the stiffness due to the combination of both the fiber and sheet directions. The constitutive law for the passive myocardium is then given by the following strain energy function:

$$
\Psi=\frac{a}{2 b}\left[\exp \left\{b\left(I_{1}-3\right)\right\}-1\right]+\sum_{i \in\{\mathrm{f}, \mathrm{s}\}} \frac{a_{i}}{2 b_{i}}\left[\exp \left\{b_{i}\left(I_{4 i}-1\right)^{2}\right\}-1\right]+\frac{a_{\mathrm{fs}}}{2 b_{\mathrm{fs}}}\left\{\exp \left(b_{\mathrm{fs}} I_{8 \mathrm{fs}}^{2}\right)-1\right\}+\frac{1}{2} K(J-1)^{2}
$$

where $I_{1}, I_{4 \mathrm{i}}$ and $I_{8 \mathrm{fs}}$ are a-priori known invariants, defined as:

$$
I_{1}=\operatorname{tr}(\mathbb{C}) \quad I_{4 \mathrm{f}}=\mathbf{f}_{0} \cdot\left(\mathbb{C} \mathbf{f}_{0}\right) \quad I_{4 \mathrm{~s}}=\mathbf{s}_{0} \cdot\left(\mathbb{C} \mathbf{s}_{0}\right) \quad I_{8 \mathrm{fs}}=\mathbf{f}_{0} \cdot\left(\mathbb{C} \mathbf{s}_{0}\right)
$$

where $\mathbb{C}$ is the right Cauchy-Green tensor, while $\mathbf{f}_{0}$ and $\mathbf{s}_{0}$ are the fibre and sheet orientations respectively. This strain energy function can then be differentiated to get the forces acting on the myocardium. By applying appropriate conservation laws, we can get equations of motion, and from there predict LV behaviour during diastole. For further details, the reader is directed to the original publication [2].

\section{Parameter Estimation}

Myocardium stiffness during the passive stage of the cardiac cycle can be used to make a thorough evaluation of LV functionality. For example, it has been shown that these stiffness levels are much higher in patients with diastolic heart failure than the levels found in healthy control subjects [7]. It is for this reason that the determination of the biophysiological parameter vector $\boldsymbol{\xi}$ is of interest, however direct measurements of the eight stiffness levels require the heart to be extracted from the patient, and therefore can only be done in post-mortem autopsy. Non-invasive procedures, such as MRI scans, are instead limited to observing patterns in the deformations of the LV during the pump-cycle. It is here that the HO law presents an opportunity for clinical use, as it can also model these deformations, given a set of parameter values. Thus, we can obtain an estimate of the parameters by finding those values which minimise the discrepancy between the quantities we extract from the MRI scans, and the corresponding outputs of the model. This requires a choice of which specific outputs should be extracted from the scans for inclusion in the minimisation procedure. In accordance with previous work [5, 6], we here concentrate on 25 outputs; LV volume at end-diastole, and circumferential strain values at 24 locations on the LV surface.

Determining $\boldsymbol{\xi}$ for noisy, real world data can be challenging. The sensitivity analysis carried out in [8] suggests that when only extracting circumferential strains and volume from the MRI scans, some parameters are only weakly identifiable. Further identifiability issues are caused by strong correlations between the stiffness values. For this reason, here we focus on parameter estimation for a reduced form of the HO law as described in [8], which makes use of the linear redundancy in $\xi$ to express it in terms of four parameters, which we denote

$$
\boldsymbol{\theta}=\left(\theta_{1}, \theta_{2}, \theta_{3}, \theta_{4}\right)^{\top}
$$

We also denote the measured quantities from the MRI, after non-dimensionalisation, by

$$
\mathbf{y}=\left(\mathrm{y}_{0}, \mathrm{y}_{1}, \ldots, \mathrm{y}_{K}\right)^{\top}
$$

where $y_{0}$ is the non-dimensionalised LV volume, and $y_{1}, \ldots, y_{24}$ are the non-dimensionalised circumferential strains, and the corresponding outputs from the $\mathrm{HO}$ model, which depend on the cardio-mechanic parameters $\boldsymbol{\theta}$ and the LV geometry, $\mathcal{H}$, as:

$$
\mathbf{f}(\boldsymbol{\theta}, \mathcal{H})=\left(\mathrm{f}_{0}(\boldsymbol{\theta}, \mathcal{H}), \mathrm{f}_{1}(\boldsymbol{\theta}, \mathcal{H}), \ldots, \mathrm{f}_{K}(\boldsymbol{\theta}, \mathcal{H})\right)^{\top}
$$


An estimate of $\boldsymbol{\theta}$ can then be found for a given LV geometry $\mathcal{H}$ by minimisation of some loss function $L$ :

$$
\hat{\boldsymbol{\theta}}=\underset{\boldsymbol{\theta}}{\arg \min } L(\boldsymbol{\theta})
$$

where a standard choice of loss is the mean squared error:

$$
L(\boldsymbol{\theta})=\frac{1}{M+1} \sum_{k=0}^{M}\left(\mathrm{y}_{k}-\mathrm{f}_{k}(\boldsymbol{\theta}, \mathcal{H})\right)^{2}
$$

While this approach appears to provide a method for non-invasive estimation of a patient's passive myocardial stiffness levels, it faces prohibitive computational costs. This is because finding a solution to (पD) requires the use of an iterative optimisation algorithm, which may require hundreds or thousands of iterations to converge. At each iteration, a forward simulation of the HO model is required, which takes several minutes on even a high performance computer. Therefore, this method is not viable for use as a real-time clinical decision support tool.

\section{Emulation}

One method which has the potential to overcome this computational burden is emulation. An emulator $\hat{\mathbf{f}}$ is a statistical surrogate model which approximates a computer simulator $\mathbf{f}$, based on a data set of input-output pairs from the simulator:

$$
\mathcal{D}=\left\{\left(\mathbf{x}_{\mathbf{i}}, \mathbf{f}\left(\mathbf{x}_{\mathbf{i}}\right), i=1,2, \ldots N\right\}\right.
$$

In the context of the HO model, the inputs are the parameter vector $\boldsymbol{\theta}$ and LV geometry $\mathcal{H}$, so we have $\mathbf{x}_{\mathbf{i}}=\left(\boldsymbol{\theta}_{i}, \mathcal{H}_{i}\right)$ above. The location of the $N$ inputs points can be chosen using a space-filling design to ensure a dense coverage in the region of interest. The advantage of emulation methods for our purposes is that we can perform these $N$ costly simulator runs and train the emulator in advance of clinical deployment. When patient data becomes available in clinic, we can replace $\mathbf{f}$ with $\hat{\mathbf{f}}$ in (पD) to obtain parameter estimates in real time, as predictions from the emulator can be made several orders of magnitude faster than predictions from the simulator.

It is important to quantify the loss in parameter estimation accuracy incurred when using the surrogate emulator in place of the simulator. This can be done by creating an independent test set of inputs and outputs for the HO law, $\mathcal{D}_{\text {test }}$ in the same manner as (9). We then estimate the input parameters for each test point by minimising the loss between the simulator outputs and the outputs of the emulator. The accuracy of these estimates can subsequently be evaluated by comparing them to the known true parameters values.

Recent research into the use of emulation methods for parameter inference in the HO law has demonstrated these methods can accurately replicate the results of the underlying simulator. Initial work demonstrated the effectiveness of independent, local GPs, one for each output of the simulator [5]. Further research then improved on these results by using a single, multivariate output GP to model all simulator outputs simultaneously [6]. Both studies above concentrated on parameter inference for the fixed LV geometry of a healthy volunteer. For practical deployment however, the emulation must also be able to account for the specific LV geometry of a patient, which will not be known in advance of their arrival in clinic. Emulation methods which can account for subject-specific geometries are thus the remit of this work.

\section{Data}

As outlined above, the LV mathematical model takes as input the material properties describing the stiffness of the myocardium, $\boldsymbol{\theta}$, and a LV mesh, $\mathcal{H}$. In order to train an accurate emulator, we need to generate a data set that has dense coverage over the space of possible configurations of parameters and LV geometries. The problem is that each LV mesh has a dimension of over 17,000. Generating a data set that has dense coverage over a space of this dimensionality is impossible. Therefore, we require a representation of the LV meshes that is low enough in dimension to make emulation possible, but that also accurately reconstructs the original meshes when projected back to the original high dimensional space. In a previous study [9], we have found that principal component analysis (PCA) provides the same reconstruction accuracy as competing methods at lower computational costs. Given the choice of PCA, deciding how many dimensions our reduced LV 
representation should consist of is equivalent to selecting the number of principal components (PCs) to use. As we use more PCs, the discrepancy falls between the outputs of the simulator when the original mesh is inputted and the outputs when the reconstruction from the PC representation is used. However, increasing the dimensionality of the LV representation makes it more difficult for the emulator to accurately replicate the results of the simulator, as it must learn a function in a higher dimensional space. With this trade-off in mind, we here opt for a representation using 5 PCs, trained on a set of 135 discretised LV geometries. From Figure 1(a) we see that with 5PCs, almost $90 \%$ of the variation in the original geometries is retained. As a result, when we project back to the original space, we obtain low reconstruction error. A sample 5PC reconstruction is shown in red in Figure 1(b), alongside the original mesh in blue.

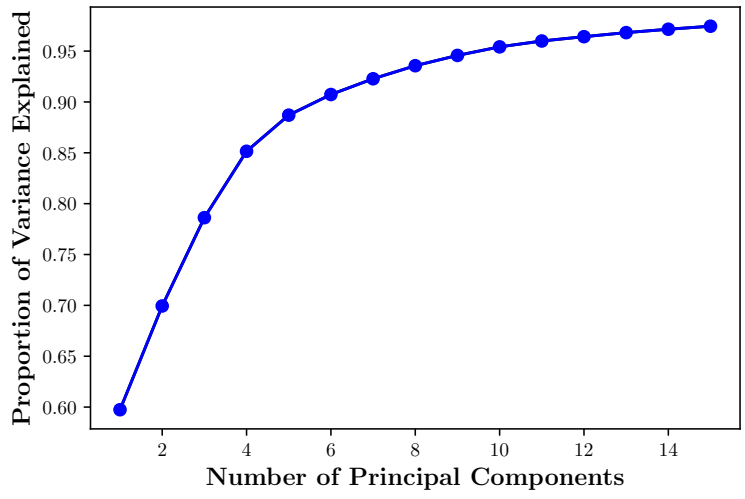

(a)

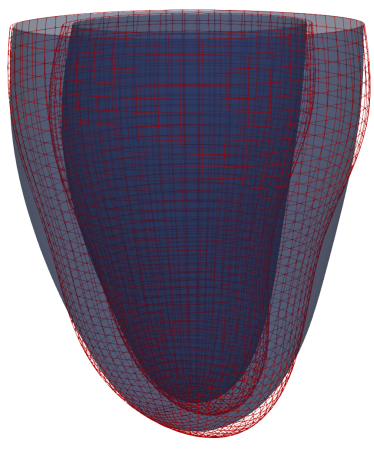

(b)

Fig. 1: For emulation, we require a low dimensional representation of the LV mesh. In (a) we plot the cumulative proportion of variation explained in the geometry data against the number of principal components. In (b) show an initial mesh (endocardium and epicardium) in blue along with a reconstruction of the mesh in red. This reconstruction is obtained by projecting into the low dimensional space and then transforming back into the initial space.

Combining the 5 dimensional PC space with the 4 dimensional material property space, we now have a 9 dimensional space to be emulated over. By using a Sobol sequence [10], we generated a batch of input points within physiological realistic boundaries, and ran forward simulations from each point. Some simulations crash, so we ran in excess of 16,000 and took the additional successful simulations as a test set. This provided a test set of size 539 .

\section{Emulation Methods}

\subsection{Gaussian Processes}

Gaussian process (GP) regression is a powerful, non-parametric Bayesian modelling technique. For a comprehensive overview, the reader can see [11]. A stochastic process is referred to as a Gaussian process if any finite subset of random variables from the process has a Gaussian distribution. A GP can be used in a Bayesian regression context by considering it as a prior directly on the space of functions of interest

$$
\mathrm{f}(\mathbf{x}) \sim \mathcal{G P}\left(\mu(\mathbf{x}), k\left(\mathbf{x}, \mathbf{x}^{\prime}\right)\right)
$$

where the mean function $\mu(\mathbf{x})$ and positive definite kernel function $k\left(\mathbf{x}, \mathbf{x}^{\prime}\right)$ completely specify the GP. For this work, we considered constant mean functions and linear mean functions respectively. For the kernel, we chose the ARD squared exponential kernel

$$
k\left(\mathbf{x}_{\mathbf{i}}, \mathbf{x}_{\mathbf{j}}\right)=\alpha^{2} \exp \left(-\frac{1}{2} \sum_{k=1}^{D} \frac{\left(x_{i k}-x_{j k}\right)^{2}}{\ell_{k}^{2}}\right)
$$

Given a set of training data, the prior GP can be updated using Bayes Theorem to a posterior distribution, which is itself Gaussian, over a collection of finite test points of interest. GPs have proved popular for the purposes of emulation, as they can 
offer exact interpolation of deterministic simulator outputs [4]. The main drawback with the GP framework is that training time grows cubicly with the number of inputs and prediction times grow quadratically. This complexity precludes the use of a standard GP model for emulation of the data set under consideration here. For this reason, we instead use three variants of the GP approach, and these are explained below.

\subsubsection{Sparse Gaussian Processes}

Sparse GPs offer an alleviation of the computational costs of full Gaussian process models. Using a set of $m$ inducement points, these methods replace the marginal likelihood with a cheaper approximation, reducing computational complexity for a dataset of size $n$ to $O\left(\mathrm{~nm}^{2}\right)$ [12]. While optimization of inducement points can proceed under different frameworks, in this paper we follow the variational approach. Here, the inducement points are treated as variational parameters and optimized alongside the kernel hyperparameters [13]. Using an inducing set of size 2000, we build 25 Gaussian process emulators for each of the model outputs. With the aim of keeping computational costs down, optimization of the material properties proceeds by first exploring the global loss function by evaluation of a massive set of material property configurations $\left(1 \times 10^{5}\right.$ were used in total). We then initialize a gradient descent optimizer from the best point to find the parameter estimate.

\subsubsection{Local Gaussian Process Regression}

Local GPs make a prediction at a test point of interest by using a GP trained on the point's $k$ nearest neighbours in the training data [14]. Since the size of this local neighbourhood can be chosen to be much smaller than the full data set, we can make significant computational savings compared to the standard GP approach. Previous work has shown that local GPs can effectively emulate the HO model, whether the local GP is calculated with respect to input space or output space [6]. The problem with using a local GP in input space is that this requires the model to be refit at each stage of the parameter optimisation. For this reason, local GPs in output space are considered here, as this neighbourhood remains the same at each iteration. Using local GPs requires a value of $k$ to be chosen. To do so, we extracted an independent validation set of 1,000 points from the training data. A number of local GP emulators with different values of $k$ were then used to infer the parameters in the validation set, trained on the remaining training data. Each emulator was composed of 25 separate local GPs, one for each output. Prediction accuracy was found to increase monotonically with $k$, however so did computation times. We therefore decided on a value of $k=500$ to balance this accuracy/efficiency trade-off.

\subsubsection{Patchwork Kriging}

Patchwork Kriging [15] is a development of the local GP approach. It first partitions the input space into $K$ regions called patches, and fits separate, but dependent, local GPs to each patch. The dependency is enforced through the introduction of a number of "stitching points" along the boundaries between regions. These stitching points are treated as fictitious data points, through which the GPs of adjoining patches are constrained to have the same mean and variance. To perform patchwork kriging, we must choose the number of patches $K$, and the number of stitching points to put along the boundary of each patch. We did this by comparing the validation set output space prediction accuracy of different emulators, each comprised of 25 patchwork GPs, trained over a grid of the different patches and stitching points combinations. We found 64 patches to be optimal, and that accuracy increased as the number of stitching points increased, but at increased computational costs. For this reason, 3 stitching points were used on each boundary, to balance accuracy and efficiency as above.

\subsection{Neural Networks}

One of the downsides of the local and sparse Gaussian process procedures outlined above is the need to ignore some training data in the model fitting process. Neural networks offer a highly flexible, but parametric, alternative to GPs. Abandoning the non parametric nature enables more efficient predictions from the trained model independent of the initial training dataset, while also allowing us to train with the full set of data [16]. If we assume a 2 hidden layer network, we must decide the number of nodes in each layer as well as the activation functions. Using a validation set of size 1000 (leaving a training set of 15000 points) we selected the network architecture leading to the best predictive performance on the held out dataset. Low computational cost of prediction from neural networks means that we can implement gradient descent from multiple start points to estimate the material properties. We use 100 local optimizers, initialized from random points and choose the best optimized parameter as our estimate. 


\subsection{Radial Basis Function Interpolation}

A radial basis function (RBF) interpolator of a function $\mathrm{f}(\mathbf{x})$ takes the form of a weighted sum of radially symmetric functions $\phi$ :

$$
\hat{\mathrm{f}}(\mathbf{x})=\sum_{i=1}^{N} \mathrm{w}_{i} \phi\left(\left\|\mathbf{x}-\mathbf{x}_{\mathbf{i}}\right\|\right),
$$

given a training data set as in (9). The weights $\mathrm{w}_{i}$ can be found as those values which give exact interpolation to the training data. A number of different choices of basis function for the RBF emulator were compared in terms of parameter estimation accuracy on the held out validation set of 1,000 points. The cubic basis function:

$$
\phi\left(\left\|\mathbf{x}-\mathbf{x}^{\prime}\right\|\right)=\left\|\mathbf{x}-\mathbf{x}^{\prime}\right\|^{3}
$$

was found to achieve the best results, so this was used for prediction on the test data set.

\subsection{Random Forests}

Random forests [17] regression is a non-parametric modelling technique which makes prediction by averaging over an ensemble of randomised decision trees. The randomness is introduced by only considering a random subset of predictors and training points respectively at each node as we grow the decision trees. While each tree grown in this manner is weakly informative, by averaging their results we can obtain strong predictions. A validation set was used to tune the size of the ensembles and level of randomness in the feature and data sampling for each output dimension respectively.

\section{Results}

We compare the seven emulation methods outlined in Section 6 based on their parameter estimation error on the independent test set of 539 points described in Section 5. These parameter estimates were found by minimising the MSE between the true simulator outputs and the outputs of the respective emulators:

$$
\hat{\boldsymbol{\theta}}_{j}=\underset{\boldsymbol{\theta}}{\arg \min }\left\|\mathbf{f}\left(\boldsymbol{\theta}_{j}, \mathcal{H}_{j}\right)-\hat{\mathbf{f}}\left(\boldsymbol{\theta}, \mathcal{H}_{j}\right)\right\|^{2}
$$

for all test points $j=1,2, . .539$ using iterative optimisation methods. We then evaluated the MSE between the true and predicted values:

$$
\operatorname{MSE}_{j}=\frac{1}{D} \sum_{k=1}^{D}\left(\theta_{j k}-\hat{\theta}_{j k}\right)^{2}
$$

where $D=4$. Following this procedure for all seven emulation methods, we obtain a list of 539 MSEs for each method respectively. The median, first quartile and third quartile of each of these lists is given in Table 1.

Table 1: Test Set Parameter Estimate Mean Squared Errors

\begin{tabular}{|c|c|c|c|}
\hline Method & $\mathbf{2 5}^{\text {th }}$ Perc. & Median & $\mathbf{7 5}^{\text {th }}$ Perc. \\
\hline Sparse GP & $3.3 \times 10^{-2}$ & $1.1 \times 10^{-1}$ & $2.5 \times 10^{-1}$ \\
\hline Local GP & $4.3 \times 10^{-3}$ & $1.6 \times 10^{-2}$ & $4.7 \times 10^{-2}$ \\
\hline Patchwork GP & $4.1 \times 10^{-2}$ & $1.3 \times 10^{-1}$ & $3.6 \times 10^{-1}$ \\
\hline Neural network & $6.6 \times 10^{-3}$ & $2.1 \times 10^{-2}$ & $6.0 \times 10^{-2}$ \\
\hline RBF & $7.2 \times 10^{-3}$ & $2.3 \times 10^{-2}$ & $8.8 \times 10^{-2}$ \\
\hline Random Forests & $4.0 \times 10^{-1}$ & $9.6 \times 10^{-1}$ & 1.85 \\
\hline
\end{tabular}

Table 1 shows that neural networks, RBFs and local GPs attained the best parameter estimation accuracy with patchwork kriging and sparse Gaussian processes each achieving slightly worse results. The sharp deterioration in accuracy attained when using the random forest emulator is of note. This was particularly due to the difficulty encountered in estimating $\theta_{3}$ and $\theta_{4}$ with random forests. These two inputs were weakly informative about the simulator outputs, and hence were less frequently 
considered for splitting on when constructing the underlying decision trees. This in turn made it difficult to precisely identify the values of $\theta_{3}$ and $\theta_{4}$ in the parameter optimisation. The best performing models also encountered difficulty in identifying the final two dimensions of $\boldsymbol{\theta}$. This can be seen in Figure 2, which plots the true values of the test set parameters versus those predicted by the local Gaussian process emulator for each of the four dimensions of $\boldsymbol{\theta}$ respectively. We can see greater deviations from the red lines, which represent perfect prediction accuracy, for $\theta_{3}$ and $\theta_{4}$ when compared to the first two dimensions of $\boldsymbol{\theta}$.

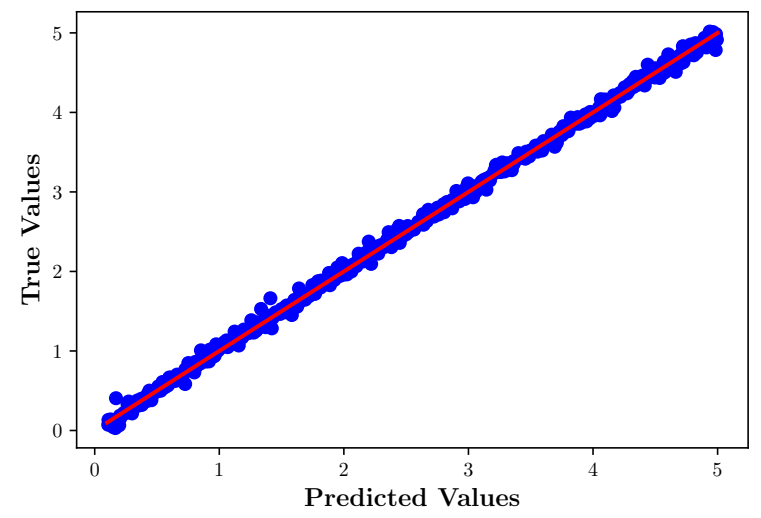

(a) $\theta_{1}$

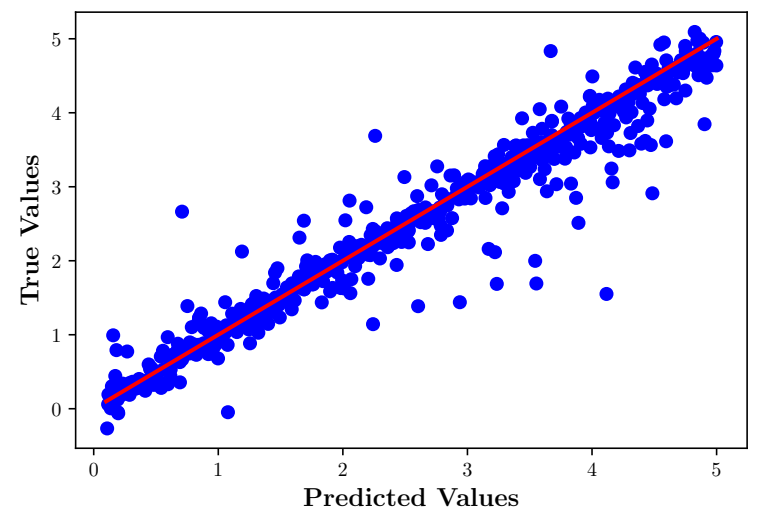

(c) $\theta_{3}$

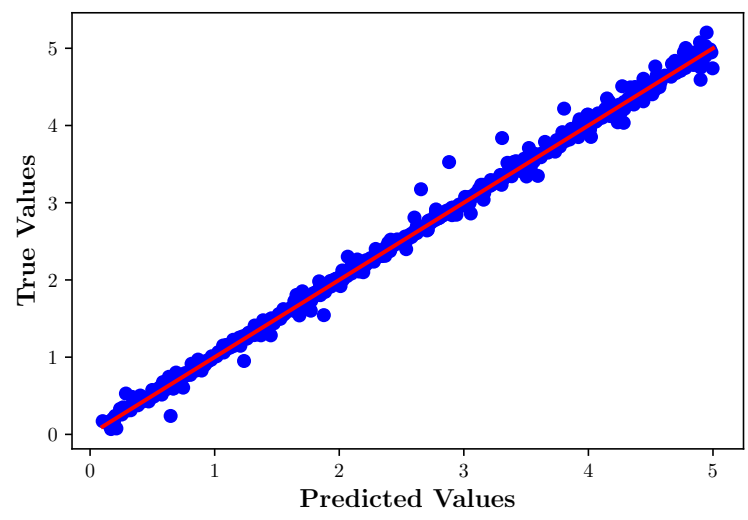

(b) $\theta_{2}$

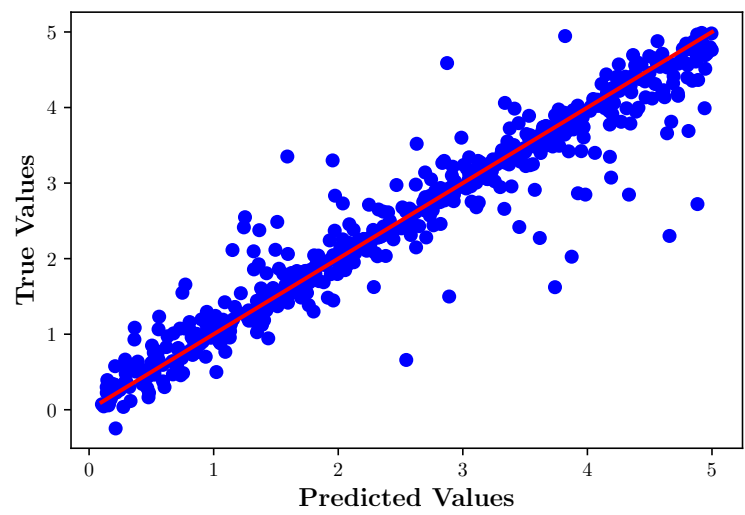

(d) $\theta_{4}$

Fig. 2: Plot of true parameters values of the test set versus those predicted by local GP for each dimension respectively. Points lying on the red lines of unit slope indicate perfect prediction accuracy.

\section{Discussion}

The results of this study are encouraging, displaying the ability of the statistical model to generalize the single geometry mapping to multiple left ventricle geometries. Current results show that for geometries spanned by the 5 principal components, $\theta_{1}$ and $\theta_{2}$ can be accurately identified given the in vivo data. Identifiability issues that did not appear in previous studies have become apparent in this multi-geometry setting, where higher errors occur in parameter estimates of $\theta_{3}$ and $\theta_{4}$. This can be explained by considering the physical representation of these parameters. In the current study end diastolic pressure (EDP) was assumed to be $8 \mathrm{mmHg}$, a value considered sensible for healthy subjects [18]. Outputs of the simulator are known to be less sensitive to $\theta_{3}$ in this pressure domain, since this parameter relates to the behaviour of the myocardium in the non-linear portion of the stress-stretch relationship. A solution to this problem that we are currently exploring is to make use of ex vivo pressure-volume data to allow us to extrapolate the behaviour of the myocardium beyond the in vivo range for each individual geometry. This information comes in the form of the Klotz curve, an empirical relationship found to exist between the pressure 
and normalized volume [19]. The issue of $\theta_{4}$ is currently more difficult to alleviate. At present, the simulator-and in vivo data-only relate to circumferential strains from the LV wall. Sensitivity of $\theta_{4}$ primarily to the radial strains means that information provided by the data about $\theta_{4}$ is limited. In the future we hope to be able to measure and simulate these strains to enable accurate inference for $\theta_{4}$.

\section{Acknowledgements}

This work was carried out as part of the SofTMech ${ }^{\text {MP }}$ project, funded by EPSRC, grant reference number EP/S030875/1. Alan Lazarus is partially funded by a scholarship from GSK. Dirk Husmeier is funded by the Royal Society of Edinburgh, grant reference number 62335 .

\section{References}

[1] V. Wang, P. Nielsen, M. Nash, "Image-based predictive modeling of heart mechanics," Rev. Biomed. Engng., vol. 17, pp. $351-383,2015$.

[2] G. A. Holzapfel and R. W. Ogden, "Constitutive modelling of passive myocardium: a structurally based framework for material characterization," Phil. Trans. R. Soc. A, vol. 367, pp. 3445-3475, 2009.

[3] H. Gao, A. Aderhold, K. Mangion, X. Luo, D. Husmeier, C. Berry, "Changes and classification in myocardial contractile function in the left ventricle following acute myocardial infarction," J. R. Soc. Interface, vol. 14, 2017.

[4] M.C. Kennedy and A. O'Hagan, "Bayesian calibration of computer models," J. R. Stat. S. B, vol. 63, pp. 425 - 464, 2001.

[5] V. Davies, N. Umberto, A. Lazarus, H. Gao, B. Macdonald, C. Berry, X. Luo, D. Husmeier, "Fast parameter inference in a biomechanical model of the left ventricle by using statistical emulation," J. R. Stat. S. C, vol. 68, pp. 1555-1576, 2019.

[6] U. Noe, A. Lazarus, H. Gao, V. Davies, B. Macdonald, K. Mangion, C. Berry, X. Luo, D. Husmeier, "Gaussian process emulation to accelerate parameter estimation in a mechanical model of the left ventricle: a critical step towards clinical end-user relevance," J. R. Soc. Interface, vol. 16, 2019.

[7] J. Xi, W. Shi, D. Rueckert, R. Razavi, N.P. Smith, P. Lamata, "Understanding the need of ventricular pressure for the estimation of diastolic biomarkers," Biomech. Modlng. Mechbiol., vol. 13, pp. 747-757, 2014.

[8] H. Gao, W. Li, L. Cai, C. Berry, X. Luo, "Parameter estimation in a Holzapfel-Ogden law for healthy myocardium," J. Engng Math., vol. 95, pp. 231-248, 2015.

[9] L. Romaszko, A. Lazarus, H. Gao, A. Borowska, X. Luo, D. Husmeier, "Massive Dimensionality Reduction for the Left Ventricular Mesh," in Proceedings of the International Conference on Statistics: Theory and Applications, Lisbon, Portugal, 2019.

[10] K. Fang, R. Li, A. Sudjianto, Design and modeling for computer experiments. London, UK: Chap. and Hall/CRC, 2006.

[11] C.E. Rasmussen and C.K.I. Williams, Gaussian processes for machine learning. Cambridge, MA: MIT Press, 2006.

[12] M. Bauer, M. van der Wilk, C.E. Rasmussen, "Understanding Probabilistic Sparse Gaussian Process Approximations," in Advances in Neural Information Processing Systems 29, pp. 1533 -1541, 2016.

[13] M. Titsias, "Variational Learning of Inducing Variables in Sparse Gaussian Processes," in Proceedings of the Twelfth International Conference on Artificial Intelligence and Statistics, Clearwater Beach, FL, 2009.

[14] R.B. Gramacy and D.W. Apley, "Local Gaussian process approximation for large computer experiments," J. Comput. Graph. Stat., vol. 24, pp. 561-578, 2015

[15] C. Park and D. Apley, "Patchwork Kriging for Large-scale Gaussian Process Regression," Journal of Machine Learning Research, vol. 19, pp. 1-43, 2018.

[16] C.M. Bishop, Neural Networks for Pattern Recognition. Oxford University Press, Inc., USA, 1995.

[17] L. Breiman, "Random Forests," Machine Learning, vol. 45, pp. 5-32, 2001.

[18] R.J. Bouchard, J.H. Gault, J. Ross Jr., "Evaluation of pulmonary arterial end-diastolic pressure as an estimate of left ventricular end-diastolic pressure in patients with normal and abnormal left ventricular performance," Circulation, vol. 44, pp. $1072-1079,1971$.

[19] S. Klotz, I. Hay, M. Dickstein, G. Yi, J. Wang, M. Maurer, D. Kass, D. Burkhoff, "Single-beat estimation of enddiastolic pressure-volume relationship: a novel method with potential for noninvasive application," American Journal of Physiology-Heart and Circulatory Physiology, 2006. 